\title{
Radiothérapie hypofractionnée pour le traitement de l'acné chéloïdienne de la nuque
}

\author{
Jennifer Yin Yee Kwan MD, May Tsao MD, Elizabeth A. Barnes MD
}

- Citation : CMAJ 2021 October 18;193:E1595. doi : 10.1503/cmaj.210307-f

Voir la version anglaise de l'article ici : www.cmaj.ca/lookup/doi/10.1503/cmaj.210307
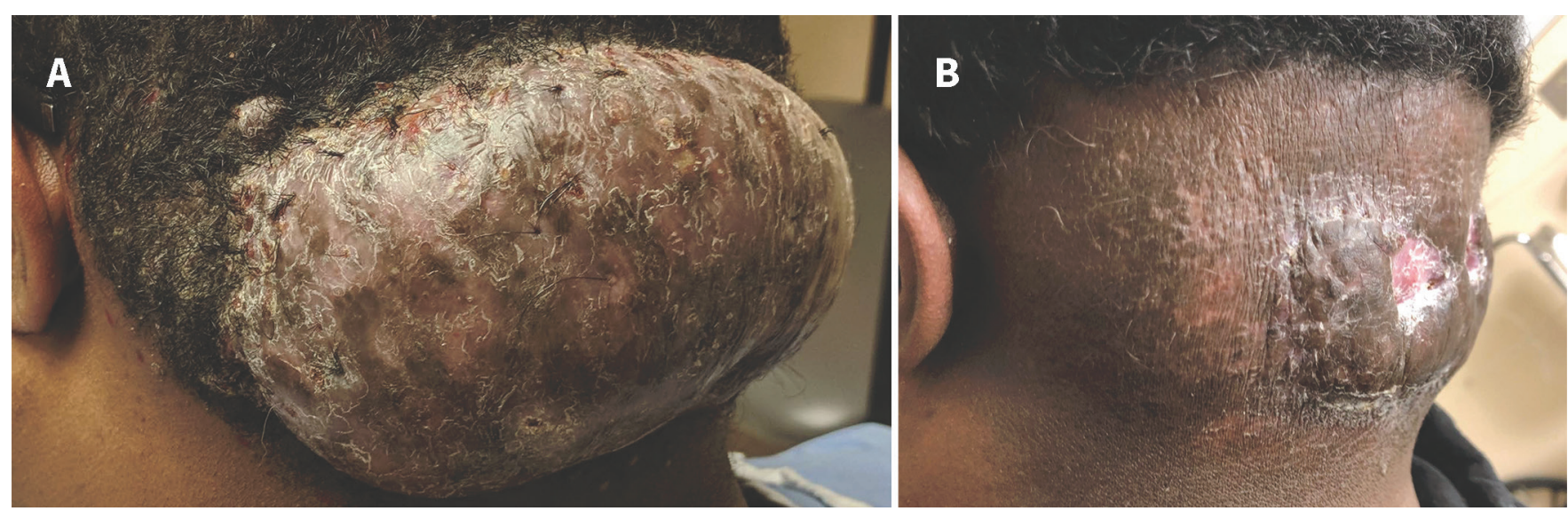

Figure 1 : Photographies d'une lésion d'acné chéloïdienne de la nuque chez un homme de 27 ans d'origine africaine (A) avant la radiothérapie et (B) un an après la radiothérapie.

$\mathbf{U}$ n homme de 27 ans d'origine africaine a consulté dans notre clinique de radiothérapie pour une lésion chéloïdienne de $15 \times 8 \times 4 \mathrm{~cm}$ à la nuque (acné chéloïdienne), récurrente depuis 5 ans (figure 1A). Les traitements antérieurs par isotrétinoïne systémique, antibiothérapie et excision chirurgicale sans radiothérapie adjuvante ont été inefficaces et le patient n'était pas candidat à une reprise de l'exérèse en raison de la taille de sa lésion. Incommodé par le caractère inesthétique de cette dernière, il a réduit ses contacts sociaux, ce qui a affecté sa capacité d'occuper un emploi.

Le patient a subi une radiothérapie radicale par arcthérapie volumétrique modulée (35 Gy en 5 fractions hebdomadaires) dans le but de réduire la taille de la lésion chéloïdienne. Après la radiothérapie, il a eu besoin de soins de plaie réguliers à mesure que la lésion régressait. À 19 mois, il ne présentait aucune récurrence (figure 1B).

L'acné chéloïdienne de la nuque est un trouble inflammatoire chronique qui prend naissance dans un follicule pileux dysfonctionnel et entraîne la formation de papules et de plaques chéloïdiennes ${ }^{1}$. Elle s'observe presque exclusivement chez les personnes d'origine africaine et principalement chez les hommes $(20: 1)^{2}$. Les estimations de sa prévalence, incluant les tableaux allant de légers à graves, sont de $0,5 \%$ à 13,6\% $\%^{2}$. L'acné chéloïdienne de la nuque est diagnostiquée cliniquement sans biopsie et les cas légers peuvent être traités au moyen de corticostéroïdes, de rétinoïdes ou d'antibiotiques ${ }^{2}$. Le traitement au laser et la photothérapie peuvent être utilisés pour réduire le nombre ou la taille des lésions ${ }^{2}$. Dans le cas de lésions fibreuses ou persistantes, l'excision est parfois utile²; par contre, la chirurgie utilisée seule est associée à un taux plus élevé de récidives ${ }^{3}$. La corticothérapie ou la radiothérapie adjuvantes peuvent contribuer à réduire les récidives, mais il faut en soupeser les risques ${ }^{4}$.

Les effets indésirables aigus de la radiothérapie incluent l'érythème cutané, la desquamation, la déhiscence de la plaie et l'infection ${ }^{4}$, tandis que ses effets indésirables tardifs incluent des modifications pigmentaires, la télangiectasie, la fibrose, l'alopécie et un risque 3 fois plus grand de néoplasie secondaire affectant la région traitée ${ }^{4,5}$. L'incidence des effets indésirables liés à la radiothérapie est de plus de $50 \% 4$.

\section{Références}

1. Salami T, Omeife H, Samuel S. Prevalence of acne keloidalis nuchae in Nigerians. Int J Dermatol 2007;46:482-4.

2. Ogunbiyi A. Acne keloidalis nuchae: prevalence, impact, and management challenges. Vol. 9. Clin Cosmet Investig Dermatol 2016;9:483-9.

3. Maranda EL, Simmons BJ, Nguyen AH, et al. Treatment of acne keloidalis nuchae: a systematic review of the literature. Dermatol Ther (Heidelb) 2016;6:363-78. 
4. Speranza G, Sultanem K, Muanza T. descriptive study of patients receiving excision and radiotherapy for keloids. Int J Radiat Oncol Biol Phys 2008;71:1465-9.

5. Lichter MD, Karagas MR, Mott LA, et al. Therapeutic ionizing radiation and the incidence of basal cell carcinoma and squamous cell carcinoma. Arch Dermatol 2000;136:1007-11.

Intérêts concurrents : Aucun déclaré.

Cet article a été révisé par des pairs.

Les auteures ont obtenu le consentement du patient.

Affiliations: Département de radio-oncologie (Kwan, Tsao, Barnes), Université de Toronto; Département de radio-oncologie (Tsao, Barnes), Centre d'oncologie Odette, Centre des sciences de la santé Sunnybrook, Toronto, Ont.

Propriété intellectuelle du contenu : Il s'agit d'un article en libre accès distribué conformément aux modalités de la licence Creative Commons Attribution (CC BY-NC-ND 4.0), qui permet l'utilisation, la diffusion et la reproduction dans tout médium à la condition que la publication originale soit adéquatement citée, que l'utilisation se fasse à des fins non commerciales (c.-à-d., recherche ou éducation) et qu'aucune modification ni adaptation n'y soit apportée. Voir : https:// creativecommons.org/licenses/by-nc-nd/4.0/deed.fr.

Correspondance : Elizabeth Barnes, toni.barnes@sunnybrook.ca

Les images cliniques sont choisies pour leur caractère particulièrement intéressant, classique ou impressionnant. Toute soumission d'image de haute résolution claire et bien identifiée doit être accompagnée d'une légende aux fins de publication. On demande aussi une brève explication (300 mots maximum) de la portée éducative des images, et des références minimales. Le consentement écrit du patient au regard de la publication doit être obtenu avant la soumission. 